Matemática

\title{
Os números híbridos de Leonardo
}

\author{
Leonardo's hybrid numbers
}

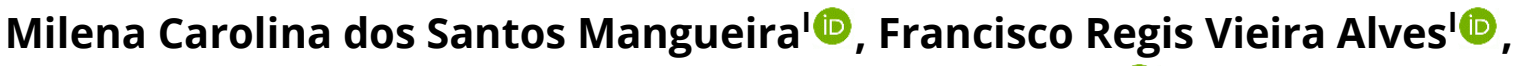 Paula Maria Machado Cruz Catarino"1(1)}

\author{
'Instituto Federal do Ceará, Fortaleza, CE, Brasil \\ " Universidade de Trás-os-Montes e Alto Douro, Portugal
}

\section{RESUMO}

No presente trabalho apresentamos um estudo sobre a hibridização da sequência de Leonardo, a partir dos resultados obtidos sobre esta sequência apresentado por Catarino e Borges (2020) e sobre o conjunto dos números híbridos, apresentado por Özdemir (2018). Ao longo do texto discutimos a hibridização da sequência de Leonardo apresentando definições, teoremas, propriedades, proposições e identidades com o intuito de apresentar novos resultados relacionados a sequência de Leonardo. $\mathrm{E}$ ainda, apresentaremos, uma relação entre os números híbridos de Leonardo com os números híbridos de Fibonacci e, a partir desta relação, exibiremos três identidades clássicas vinculadas a esta sequência híbrida de Leonardo, que são as identidades de: Catalan, Cassini e d'Ocagne.

Palavras-chave: Números híbridos; sequência de Leonardo; número híbrido de Leonardo; identidades

\section{ABSTRACT}

In the present work we present a study on the hybridization of the Leonardo sequence, from the results obtained on this sequence presented by Catarino and Borges (2020) and on the set of hybrid numbers, presented by Özdemir (2018). Throughout the text we discussed the hybridization of the Leonardo sequence presenting definitions, theorems, properties, propositions and identities in order to present new results related to the Leonardo sequence. And yet, we will present a relationship between the hybrid numbers of Leonardo with the hybrid numbers of Fibonacci and, from this relationship, we will display three classic identities linked to this hybrid sequence of Leonardo, which are the identities of: Catalan, Cassini and d'Ocagne.

Keywords: Hybrid numbers; Leonardo sequence; Leonardo hybrid number; identities 


\section{INTRODUÇÃO}

A sequência de Leonardo vem sendo trabalhada recentemente na literatura matemática, historicamente Alves et al. (2020) relata que não se sabe quem criou esta sequência, porém acredita-se ter sido Leonardo de Pisa (1180-1250) devido a grande semelhança com a sequência de Fibonacci e por ter o mesmo nome do matemático italiano. Esta sequência foi apresentada inicialmente por Catarino e Borges (2020) e é formada pelos termos $1,1,3,5,9,15 \ldots$ e gerada pela recorrência $\mathrm{Le}_{\mathrm{n}}+1=\mathrm{Le}_{\mathrm{n}}+\mathrm{Le}_{\mathrm{n}-1}+1$. E podemos encontrar estudos em torno desta sequência nos trabalhos de Alves e Vieira (2019); Shannon (2019); Vieira et al. (2019, 2020).

Por outro lado, iremos abordar o estudo do novo conjunto de números, os Números Híbridos, onde será apresentado, de forma sucinta. Com isso, será realizado a hibridização da sequência de Leonardo, ou seja, associar os números híbridos a sequência de Leonardo. A hibridização de sequências recorrentes vem sendo abordada recentemente em alguns trabalhos da matemática pura, tais como: Catarino (2019); Cerda-Morales (2018); Szynal-Liana (2018); Szynal-Liana e Włoch (2019); Mangueira et al. (2020a,b); Mangueira e Alves (2020).

Com isso, neste artigo tem-se o intuito de explorar a sequência de Leonardo realizando a sua hibridização. Assim, a seguir, iremos definir os números híbridos de Leonardo, mostraremos a recorrência da sequência híbrida de Leonardo e sua extensão para os números de índices inteiros não positivos. E ainda, a equação característica, o conjugado, caráter, norma, vetor representação do número híbrido de Leonardo, o vetor híbrido, o inverso desse número híbrido, forma matricial, função geradora, a fórmula de Binet e algumas identidades clássicas, são elas: Catalan, Cassini e d'Ocagne. 


\section{PRELIMINARES}

\subsection{Números Híbridos}

Um novo conjunto numérico foi apresentado por Özdemir (2018), onde ele estudou três sistemas numéricos juntos, sejam eles: complexos, hiperbólicos e duais estando combinados um com outro. Assim, ele denominou esse novo número como número híbrido, denotado por K. A partir disso, serão definidos os números híbridos, alguns teoremas, propriedades e a sua forma matricial.

Definição 2.1

Um número híbrido é definido como:

$K=\left\{z=a+b i+c \varepsilon+d h: a, b, c, d \in R, i^{2}=-1, \varepsilon^{2}=0, h^{2}=1, i h=-h i=\varepsilon+i\right\}$

E ainda, pode-se efetuar algumas propriedades e operações com os números híbridos, sendo elas:

Tomando dois números híbridos $\mathrm{z}_{1}=\mathrm{a}_{1}+\mathrm{b}_{1} \mathrm{i}+\mathrm{c}_{1} \varepsilon+\mathrm{d}_{1} \mathrm{~h}$ e $\mathrm{z}_{2}=\mathrm{a}_{2}+\mathrm{b}_{2} \mathrm{i}+\mathrm{c}_{2} \varepsilon+$ $\mathrm{d}_{2} \mathrm{~h}$ e $\mathrm{s} \in \mathrm{R}$ obtemos:

- Igualdade: $\mathrm{z}_{1}=\mathrm{z}_{2}$, se e somente se, $\mathrm{a}_{1}=\mathrm{a}_{2}, \mathrm{~b}_{1}=\mathrm{b}_{2}, \mathrm{c}_{1}=\mathrm{c}_{2}$ e $\mathrm{d}_{1}=\mathrm{d}_{2}$

- Soma $z_{1}+z_{2}=\left(a_{1}+a_{2}\right)+\left(b_{1}+b_{2}\right) i+\left(c_{1}+c_{2}\right) \varepsilon+\left(d_{1}+d_{2}\right) h$

- Subtração $z_{1}-z_{2}=\left(a_{1}-a_{2}\right)+\left(b_{1}-b_{2}\right) i+\left(c_{1}-c_{2}\right) \varepsilon+\left(d_{1}-d_{2}\right) h$

- Multiplicação por escalar s.z = s.a + s.bi + s.ce + s.dh

O produto híbrido é obtido distribuindo-se os termos à direita, preservando a ordem de multiplicação das unidades e depois escrevendo os valores seguintes, substituindo cada produto de unidades pelas igualdades $\mathrm{i}^{2}=-1, \varepsilon^{2}=0, \mathrm{~h}^{2}=1$, ih $=$ $-h i=\varepsilon+i$. Usando essas identidades, podemos encontrar o produto de quaisquer duas unidades híbridas. E ainda podemos apresentar a tabela da multiplicação de um número híbrido, como mostra a Tabela 1. 
Tabela 1 - Tabela de multiplicação para um número híbrido

\begin{tabular}{c|cccc}
\hline & 1 & $\mathrm{i}$ & $\varepsilon$ & $\mathrm{h}$ \\
\hline 1 & 1 & $\mathrm{i}$ & $\varepsilon$ & $\mathrm{h}$ \\
$\mathrm{i}$ & $\mathrm{i}$ & -1 & $1-\mathrm{h}$ & $\varepsilon+\mathrm{i}$ \\
$\varepsilon$ & $\varepsilon$ & $1+\mathrm{h}$ & 0 & $-\varepsilon$ \\
$\mathrm{h}$ & $\mathrm{h}-\varepsilon$ & $-\mathrm{i}$ & $\varepsilon$ & 1 \\
\hline
\end{tabular}

Fonte: Autores (2021)

A operação de multiplicação nos números híbridos não é comutativa, mas tem a propriedade de associatividade e o conjunto K dos números híbridos forma um anel não comutativo.

E ainda, temos o conjugado de um número híbrido $\mathrm{z}=\mathrm{a}+\mathrm{bi}+\mathrm{c} \varepsilon+\mathrm{dh}$, denotado por $\bar{z}$, é definido como

$\bar{z}=\mathrm{a}-\mathrm{bi}-\mathrm{c} \varepsilon-\mathrm{dh}$,

e o número real $C(z)=\mathrm{z} \bar{z}=\bar{z} z=\mathrm{a}^{2}+(\mathrm{b}-\mathrm{c})^{2}-\mathrm{c}^{2}-\mathrm{d}^{2}=\mathrm{a}^{2}+\mathrm{b}^{2}-2 \mathrm{bc}-\mathrm{d}^{2}$ é chamado de caráter do número híbrido, onde a raiz do valor absoluto desse número real será a norma do número híbrido $z$, assim temos que: $\|z\|=\sqrt{|C(z)|}$.

\subsection{Sequência de Leonardo}

A sequência de Leonardo é discutida em Catarino e Borges (2020), onde é apresentada como uma sequência recorrente de números inteiros que está relacionado a sequência de Fibonacci e de Lucas que podem ser aplicadas em quase todos os campos da ciência.

A sequência de Leonardo, com $n \in \mathbb{N}$, corresponde a seguinte relação de recorrência:

$$
\mathrm{Le}_{\mathrm{n}}=\mathrm{Le}_{\mathrm{n}-1}+\mathrm{Le}_{\mathrm{n}-2}+1, \mathrm{n} \geq 2 .
$$


Para $n+1$ podemos reescrever essa relação de recorrência como $\operatorname{Le}_{n+1}=\operatorname{Le}_{n}$ $+\operatorname{Le}_{n-1}+1$.

Podemos subtrair $\mathrm{Le}_{\mathrm{n}}-\mathrm{Le}_{\mathrm{n}+1}$ e assim obter uma outra relação de recorrência equivalente para esta sequência. Observe:

$$
\begin{aligned}
& \text { Len }- \text { Len }+1=\text { Len }-1+\text { Len }-2+1-\text { Len }- \text { Len }-1-1 \\
& \text { Len }+1=2 \text { Len }- \text { Len }-2 \text {, }
\end{aligned}
$$

com as condições iniciais $\mathrm{Le}_{0}=1, \mathrm{Le}_{1}=1$.

E ainda, Catarino e Borges (2020) apresenta uma relação entre a sequência de Fibonacci e a sequência Leonardo, com $n \in \mathbb{N}$. Assim, tem-se que para $\mathrm{n} \geq 0$, é expressa por:

$$
\mathrm{Le}_{\mathrm{n}}=2 \mathrm{~F}_{\mathrm{n}+1}-1
$$

Em Catarino e Borges (2020), pode-se encontrar algumas propriedades envolvendo essa sequência, incluindo a fórmula Binet e a função geradora. Além disso, a identidade da Cassini, a identidade de Catalan e a identidade de d'Ocagne para esta sequência, também algumas expressões de somas e produtos envolvendo os termos desta sequência.

Motivado no trabalho de Özdemir (2018), e baseado na importância dessa sequência aplicaremos a definição dos números híbridos nessa sequência e nas próximas seções apresentaremos os números híbridos de Leonardo.

\section{RESULTADOS PRINCIPAIS}

Nesta seção vamos definir os números híbridos de Leonardo e alguns resultados obtidos a partir dessa definição.

\section{Definição 3.1}


O número híbrido de Leonardo, denominado por $H_{L e}+1$ é definido como:

$H_{L e}=L_{n}+L_{n}+1 i+L_{n}+2 \varepsilon+L_{n}+3 h$.

\section{Proposição 1}

A sequência dos números híbridos de Leonardo, para $\mathrm{n} \geq 2$ e com $n \in \mathbb{N}$, satisfazem as recorrências:

$$
\text { HLe }_{n}=\text { HLe }_{n-1}+\operatorname{HLe}_{n-2}+(1+i+\varepsilon+h)
$$

e

$$
\mathrm{HLe}_{\mathrm{n}+1}=2 \mathrm{HLe}_{\mathrm{n}}-\mathrm{HLe}_{\mathrm{n}-2}
$$

\section{Demonstração}

Para a Equação 1 tem-se que:

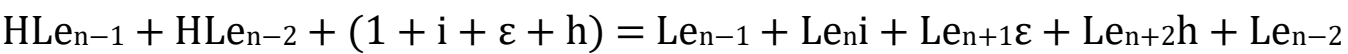

$$
\begin{aligned}
& +\mathrm{Le}_{\mathrm{n}-1} \mathrm{i}+\mathrm{Le}_{\mathrm{n}} \varepsilon+\mathrm{Le}_{\mathrm{n}+1} \mathrm{~h}+(1+\mathrm{i}+\varepsilon+\mathrm{h}) \\
& =\left(\operatorname{Len}_{\mathrm{n}-1}+\mathrm{Le}_{\mathrm{n}-2}+1\right)+\left(\operatorname{Le}_{\mathrm{n}}+\mathrm{Le}_{\mathrm{n}-1}+1\right) \mathrm{i} \\
& +\left(\operatorname{Le}_{\mathrm{n}+1}+\operatorname{Le}_{\mathrm{n}}+1\right) \varepsilon+\left(\operatorname{Le}_{\mathrm{n}+2}+\operatorname{Le}_{\mathrm{n}+1}+1\right) \mathrm{h} \\
& =\mathrm{Le}_{\mathrm{n}}+\mathrm{Le}_{\mathrm{n}+1} \mathrm{i}+\mathrm{Le}_{\mathrm{n}+2 \varepsilon}+\mathrm{Le}_{\mathrm{n}+3 \mathrm{~h}} \\
& =\text { HLen }_{\mathrm{n}}
\end{aligned}
$$

Para a Equação 2 tem-se que:

$$
\begin{aligned}
& 2 \mathrm{HLe}_{\mathrm{n}}-\mathrm{HLe}_{\mathrm{n}-2}=2\left(\operatorname{Le}_{\mathrm{n}}+\mathrm{Le}_{n+1} \mathrm{i}+\mathrm{Le}_{\mathrm{n}+2 \varepsilon}+\mathrm{Le}_{\mathrm{n}+3} \mathrm{~h}\right)-\left(\operatorname{Le}_{\mathrm{n}-2}+\operatorname{Le}_{\mathrm{n}-1} \mathrm{i}+\mathrm{Le}_{\mathrm{n}} \varepsilon+\mathrm{Le}_{\mathrm{n}+1} \mathrm{~h}\right) \\
& =\left(2 \operatorname{Le}_{n}-\operatorname{Le}_{n}-2\right)+\left(2 \operatorname{Le}_{n+1}-\operatorname{Le}_{n}-1\right) i+\left(2 L_{n}+2-L_{n}\right) \varepsilon \\
& +\left(2 \mathrm{Le}_{\mathrm{n}+3}-\mathrm{Le}_{\mathrm{n}+1}\right) \mathrm{h} \\
& =L_{n}+1+L_{n}+2 i+L e_{n}+3 \varepsilon+L_{n}+4 h \\
& =\text { HLen }_{\mathrm{n}}
\end{aligned}
$$

E ainda, estendendo esses números para índices inteiros não positivos, tem-se: 


\section{Definição 3.2}

A relação de recorrência para os números com índices negativos da sequência híbrida de Leonardo, $\mathrm{n} \geq 1$ e $n \in \mathbb{N}$, é definida por:

HLe $_{-n}=2 \mathrm{HLe}_{-\mathrm{n}+2}-\mathrm{HLe}_{-\mathrm{n}+3}$

Com os seguintes termos iniciais: $\mathrm{HLe}_{0}=1+i+3 \varepsilon+5$ h e HLe $3=3+5 i+9 \varepsilon+15$ h.

Podemos relacionar os números híbridos de Leonardo com Fibonacci, onde os números híbridos de Fibonacci foram apresentados em Cerda-Morales (2018).

Proposição 2

Para $n \geq 0$ e com $n \in \mathbb{N}$, tem-se a relação entre os números híbridos de Leonardo com os números híbridos de Fibonacci.

$$
\mathrm{HLe}_{n}=2 \mathrm{HF}_{\mathrm{n}+1}-(1+\mathrm{i}+\varepsilon+\mathrm{h}) \text {. }
$$

\section{Demonstração}

Vamos provar essa proposição por indução. Para $\mathrm{n}=0$ :

$$
\begin{aligned}
\text { HLe }_{0} & =2 \mathrm{HF}_{1}-(1+\mathrm{i}+\varepsilon+\mathrm{h}) \\
& =2(1+\mathrm{i}+2 \varepsilon+3 \mathrm{~h})-(1+\mathrm{i}+\varepsilon+\mathrm{h}) \\
& =1+\mathrm{i}+3 \varepsilon+5 \mathrm{~h}
\end{aligned}
$$

Supondo que a igualdade é válida para todo $1<\mathrm{k} \leq \mathrm{n}$, temos $\mathrm{HLe}_{\mathrm{k}}=2 \mathrm{HF}_{\mathrm{k}+1}$ $-(1+i+\varepsilon+h)$. Agora vamos provar que é válido para $k+1$, utilizando a relação de recorrência 1 e hipótese de indução, obtemos: 


$$
\begin{aligned}
\text { HLe }_{\mathrm{k}+1} & =\text { HLe }_{\mathrm{k}}+\mathrm{HLe}_{\mathrm{k}-1}+(1+\mathrm{i}+\varepsilon+\mathrm{h}) \\
& =\left[2 \mathrm{HF}_{\mathrm{k}+1}-(1+\mathrm{i}+\varepsilon+\mathrm{h})\right]+\left[2 \mathrm{HF}_{\mathrm{k}}-(1+\mathrm{i}+\varepsilon+\mathrm{h})\right]+(1+\mathrm{i}+\varepsilon+\mathrm{h}) \\
& =2\left(\mathrm{HF}_{\mathrm{k}+1}+\mathrm{HF}_{\mathrm{k}}\right)-(1+\mathrm{i}+\varepsilon+\mathrm{h}) \\
& =2 \mathrm{HF}_{\mathrm{k}+2}-(1+\mathrm{i}+\varepsilon+\mathrm{h})
\end{aligned}
$$

Com isso, provamos que a proposição é válida.

De acordo com as relações de recorrência, $\mathrm{HLe}_{\mathrm{n}+1}=2 \mathrm{HLe}_{\mathrm{n}}-\mathrm{HLe}_{\mathrm{n}-2}$, pode-se apresentar sua equação característica, por meio da relação

$$
\frac{\mathrm{H} L e_{n+1}}{\mathrm{H} L e_{n}}=\frac{H L e_{n-2}}{\mathrm{H} L e_{n}} .
$$

Utilizando o raciocínio realizado por Koshy (2001), conjecturamos que a sequência dos quocientes $\frac{\mathrm{H} L e_{n+1}}{\mathrm{H} L e_{n}}$ converge para um número positivo. Assim, temse que $\frac{\mathrm{H} L e_{n+1}}{\mathrm{H} L e_{n}}=2-\frac{\mathrm{H} L e_{n-2}}{\mathrm{H} L e_{n}} \cdot \frac{\mathrm{H} L e_{n-1}}{\mathrm{H} L e_{n-1}} \therefore \frac{\mathrm{H} L e_{n+1}}{\mathrm{H} L e_{n}}=2-\frac{1}{\frac{\mathrm{H} L e_{n-1}}{\mathrm{H} L e_{n}-2} \cdot \frac{\mathrm{H} L e_{n}}{\mathrm{H} L e_{n}-1}}$.

Denotando $x_{n}=\frac{\mathrm{H} L e_{n+1}}{\mathrm{H} L e_{n}}$, tem-se: $x_{n-1}=\frac{\mathrm{H} L e_{n}}{\mathrm{H} L e_{n}-1}$ e $x_{n-2}=\frac{\mathrm{H} L e_{n-1}}{\mathrm{H} L e_{n}-2}$. Deteminando a equação $x_{n}=2+\frac{1}{x_{n-1} \cdot x_{n-2}}$. Note que a sequência é monótona limitada, assim passando o limite e fazendo $\mathrm{n}$ tender para o infinito nesta última expressão, temse:

$$
\begin{aligned}
& \lim _{n \rightarrow \infty} x_{n}=2-\frac{1}{\lim _{n \rightarrow \infty} x_{n-1} \cdot \lim _{n \rightarrow \infty} x_{n-2}} \\
& x=2-\frac{1}{x^{2}} \\
& x^{3}-2 x^{2}+1=0
\end{aligned}
$$

onde é uma equação cúbica possuindo três raízes, sendo duas iguais as raízes da equação característica da sequência de Fibonacci e uma igual a 1.

Baseado no que já foi apresentado anteriormente pode-se apresentar o seu conjugado, seu caráter e a norma de um número híbrido de Leonardo. 


\section{Definição 3.3}

O conjugado do número híbrido de Leonardo é definido por:

$\overline{H L e_{n}}=L e_{n}-L e_{n+1} i-L e_{n+2} \varepsilon-L e_{n+3} h$.

O produto de um número híbrido pelo seu conjugado é denominado caráter do número híbrido.

\section{Definição 3.4}

O caráter do número híbrido de Leonardo é definido por:

$$
C\left(\operatorname{HLe}_{n}\right)=\operatorname{Le}_{\mathrm{n}}^{2}+\left(\operatorname{Le}_{\mathrm{n}+1}-\mathrm{Le}_{\mathrm{n}+2}\right)^{2}-\mathrm{Le}_{\mathrm{n}+2}^{2}-\mathrm{Le}^{2}{ }_{\mathrm{n}+3} .
$$

A norma de um número híbrido é gerado a partir da raiz quadrada do módulo do caráter de um número híbrido.

\section{Definição 3.5}

A norma de um número híbrido de Leonardo é definido como:

$$
\left\|H L e_{n}\right\|^{2}=\left|L e^{2}{ }_{n+1}-2 L e_{n+1} L e_{n+2}+4 L e_{n+2} L e_{n}-4 L e^{2}{ }_{n+2}\right| .
$$

Definição 3.6

Para o número híbrido de Leonardo, tem-se que o vetor representação desse número é definido por:

$$
\mathbb{V} H L e_{n}=\left(L e_{n},\left(L e_{n+1}-L e_{n+2}\right), L e_{n+2}, L e_{n+3}\right)
$$

\section{Definição 3.7}

O vetor híbrido de Leonardo é um vetor do espaço de Minkowski $\mathrm{E}^{3}{ }_{1}$ e é definido por: 


$$
\xi \mathrm{HLe}_{\mathrm{n}}=\left(\mathrm{Le}_{\mathrm{n}+1}-\mathrm{Le}_{\mathrm{n}+2}, \mathrm{Le}_{\mathrm{n}+2}, \mathrm{Le}_{\mathrm{n}+3}\right) .
$$

\section{Definição 3.8}

O inverso do número híbrido de Leonardo, com $\left\|H L e_{n}\right\| \neq 0$, é dado por:

$$
H L e_{n}^{-1}=\frac{\overline{H L e_{n}}}{C\left(H L e_{n}\right)} .
$$

Além disso, os números híbridos de Leonardo podem ser representados de forma matricial, a partir de uma matriz $2 \times 2$.

Proposição 3

Uma matriz do número híbrido de Leonardo, com $\mathrm{n} \in \mathrm{N}$, é dada por:

$$
\emptyset H L e_{n}=\left[\begin{array}{cc}
L e_{n}+L e_{n+2} & L e_{n+1}+L e_{n+2}-L e_{n} \\
3 L e_{n+2}-L e_{n}-L e_{n+1} & L e_{n}-L e_{n+2}
\end{array}\right]
$$

Demonstração

$$
\begin{aligned}
\varnothing H L e_{n} & =L e_{n}\left[\begin{array}{ll}
1 & 0 \\
0 & 1
\end{array}\right]+L e_{n+1}\left[\begin{array}{cc}
0 & 1 \\
-1 & 0
\end{array}\right]+L e_{n+2}\left[\begin{array}{cc}
1 & -1 \\
1 & -1
\end{array}\right]+L e_{n}\left[\begin{array}{ll}
0 & 1 \\
1 & 0
\end{array}\right] \\
& =\left[\begin{array}{cc}
L e_{n}+L e_{n+2} & L e_{n+1}-L e_{n+2}+L e_{n+3} \\
L e_{n+2}-L e_{n+1}+L e_{n+3} & L e_{n}-L e_{n+2}
\end{array}\right] \\
& =\left[\begin{array}{cc}
L e_{n}+L e_{n+2} & L e_{n+1}-L e_{n+2}+2 L e_{n+2}-L e_{n} \\
L e_{n+2}-L e_{n+1}+2 L e_{n+2}-L e_{n} & L e_{n}-L e_{n+2}
\end{array}\right] \\
& =\left[\begin{array}{cc}
L e_{n}+L e_{n+2} & L e_{n+1}+L e_{n+2}-L e_{n} \\
3 L e_{n+2}-L e_{n}-L e_{n+1} & L e_{n}-L e_{n+2}
\end{array}\right]
\end{aligned}
$$

Proposição 4

Se $\varnothing H L e_{n}$ corresponde a matriz hibrida do número híbrido de Leonardo, então $C\left(L e_{n}\right)=\operatorname{det}\left(\varnothing H L e_{n}\right)$.

\section{Demonstração}

$$
\operatorname{det}\left(\varnothing H L e_{n}\right)=\left|\begin{array}{cc}
L e_{n}+L e_{n+2} & L e_{n+1}+L e_{n+2}-L e_{n} \\
3 L e_{n+2}-L e_{n}-L e_{n+1} & L e_{n}-L e_{n+2}
\end{array}\right|
$$




$$
\begin{aligned}
= & \operatorname{Le}_{n}^{2}-\operatorname{Le}_{n+2}^{2}-3 \operatorname{Le}_{n+2} \operatorname{Le}_{n+1}+\operatorname{Le}_{n+1} \operatorname{Le}_{n}+\operatorname{Le}_{n+1}{ }_{n+1}-3 \operatorname{Le}_{n+2}{ }_{n+2} \\
& +\operatorname{Le}_{n} \operatorname{Le}_{n+2}+\operatorname{Le}_{n+1} \operatorname{Le}_{n+2}+3 \operatorname{Le}_{n+2} \operatorname{Le}_{n}-\operatorname{Le}_{n}{ }_{n}-\operatorname{Le}_{n} \operatorname{Le}_{n+1} \\
= & \operatorname{Le}_{n}{ }_{n}-\operatorname{Le}_{n+2}^{2}-2 \operatorname{Le}_{n+1} \operatorname{Le}_{n+2}+\operatorname{Le}_{n+1}{ }_{n+1}-3 \operatorname{Le}_{n+2}{ }_{n+2} \\
& +4 \operatorname{Le}_{n} \operatorname{Le}_{n+2}-\operatorname{Le}_{n}{ }_{n} \\
= & \operatorname{Le}^{2}{ }_{n+1}-2 \operatorname{Le}_{n+1} \operatorname{Le}_{n+2}+4 \operatorname{Le}_{n} \operatorname{Le}_{n+2}-4 \operatorname{Le}_{n+2}^{2} \\
= & C\left(\operatorname{Le}_{n}\right) .
\end{aligned}
$$

A seguir, forneceremos a função geradora de HLe ${ }_{n}$, sua fórmula de Binet e algumas identidades encontradas que envolvem essa sequências.

\section{Teorema 3.9}

A função geradora do número híbrido de Leonardo, denotado por GHLen (t), é:

$$
G_{H L e_{n}}(t)=\frac{\left(H L e_{0}+H L e_{1} t\right)(1-2 t)+H L e_{2} t^{2}}{1-2 t+t^{3}}
$$

\section{Demonstração}

Para definir a função geradora do número híbrido de Leonardo, denotado por $G_{H L e_{n}}(t)$, vamos escrever uma soma formal, em que cada parcela dessa soma tem como coeficiente um elemento da sequência dos números híbridos de Leonardo.

$$
G_{H L e_{n}}(t)=\sum_{n=0}^{\infty} H L e_{n} t^{n}
$$

Fazendo manipulações algébricas devido a relação de recorrência podemos escrever essa sequência como:

$$
\begin{aligned}
G_{H L e_{n}}(t) & =H L e_{0}+H L e_{1} t+H L e_{2} t^{2}+\sum_{n=3}^{\infty} H L e_{n} t^{n} \\
= & H L e_{0}+H L e_{1} t+H L e_{2} t^{2}+\sum_{n=3}^{\infty}\left(2 H L e_{n-1}-H L e_{n-3}\right) t^{n}
\end{aligned}
$$




$$
\begin{aligned}
& =H L e_{0}+H L e_{1} t+H L e_{2} t^{2}+2 t \sum_{n=3}^{\infty} H L e_{n-1} t^{n-1}-t^{3} \sum_{n=3}^{\infty} H L e_{n-3} t^{n-3} \\
& =H L e_{0}+H L e_{1} t+H L e_{2} t^{2}+2 t \sum_{n=0}^{\infty}\left(H L e_{n} t^{n}-H L e_{0}-H L e_{1} t-H L e_{2} t^{2}\right)-t^{3} \sum_{n=0}^{\infty} H L e_{n} t^{n} \\
& =H L e_{0}+H L e_{1} t+H L e_{2} t^{2}-2 H L e_{0} t-2 H L e_{1} t^{2}-2 H L e_{2} t^{3}+2 t \sum_{n=0}^{\infty} H L e_{n} t^{n}-t^{3} \sum_{n=0}^{\infty} H L e_{n} t^{n} \\
& =H L e_{0}+H L e_{1} t+H L e_{2} t^{2}-2 H L e_{0} t-2 H L e_{1} t^{2}-2 H L e_{2} t^{3}+2 t G_{H L e_{n}}-t^{3} G_{H L e_{n}}
\end{aligned}
$$

Assim, temos:

$$
\begin{aligned}
G_{H L e_{n}}(t)-2 t G_{H L e_{n}}+t^{3 G_{H L e_{n}}} & =H L e_{0}+H L e_{1} t+H L e_{2} t^{2}-2 H L e_{0} t-2 H L e_{1} t^{2}-2 H L e_{2} t^{3} \\
G_{H L e_{n}}(t)\left(1-2 t+t^{3}\right) & =H L e_{0}(1-2 t)+H L e_{1} t(1-2 t)+H L e_{2} t^{2}(1-2 t) \\
\Rightarrow G_{H L e_{n}}(t) & =\frac{\left(H L e_{0}+H L e_{1} t\right)(1-2 t)+H L e_{2} t^{2}}{1-2 t+t^{3}}
\end{aligned}
$$

Agora iremos explorar a existência de uma fórmula explícita para o cálculo do n-ésimo termo da sequência, sem depender da recorrência utilizando a fórmula de Binet, onde é necessário utilizar as raízes da equação característica desta sequência.

\section{Teorema 3.10}

Para $n \geq 0$, tem-se que a fórmula de Binet para o número híbrido de Leonardo é:

$$
H L e_{n}=\frac{x_{1}\left[2\left(H F_{0}-H F_{1} x_{2}\right) x_{1}^{n}-(1+i+\varepsilon+h)\right]+x_{2}\left[(1+i+\varepsilon+h)-2\left(H F_{1}-H F_{0} x_{1}\right) x_{2}^{n}\right.}{x_{1}-x_{2}},
$$

onde $x_{1}=\frac{1+\sqrt{5}}{2}$ e $x_{2}=\frac{1-\sqrt{5}}{2}$ são as raízes da equação característica da sequência.

Demonstração

A partir da fórmula de Binet dos números híbridos de Fibonacci apresentado por Cerda-Morales (2018), e utilizando a proposição 2, tem-se: 


$$
\begin{aligned}
H L e_{n} & =2\left(\frac{\left.H F_{0}-H F_{1} x_{2}\right) x_{1}^{n+1}-\left(H F_{1}-H F_{0} x_{1}\right) x_{2}^{n+1}}{x_{1}-x_{2}}\right)-(1+i+\varepsilon+h) \\
= & \frac{2\left(H F_{0}-H F_{1} x_{2}\right) x_{1}^{n+1}-2\left(H F_{1}-H F_{0} x_{1}\right) x_{2}^{n+1}-(1+i+\varepsilon+h)\left(x_{1}-x_{2}\right)}{x_{1}-x_{2}} \\
= & \frac{2\left(H F_{0}-H F_{1} x_{2}\right) x_{1}^{n+1}-x_{1}(1+i+\varepsilon+h)-2\left(H F_{1}-H F_{0} x_{1}\right) x_{2}^{n+1}+x_{2}(1+i+\varepsilon+h) x_{1}-x_{2}}{x_{1}-x_{2}} \\
= & \frac{x_{1}\left[2\left(H F_{0}-H F_{1} x_{2}\right) x_{1}^{n}-(1+i+\varepsilon+h)\right]+x_{2}\left[(1+i+\varepsilon+h)-2\left(H F_{1}-H F_{0} x_{1}\right) x_{2}^{n}\right]}{x_{1}-x_{2}}
\end{aligned}
$$

A partir da relação dos números híbridos de Fibonacci com os números híbridos de Leonardo podemos encontrar interessantes identidades, como: a identidade de Catalan, Cassini e d'Ocagne. Assim, apresentaremos a seguir, a dedução dessas identidades vinculadas ao modelo híbrido de Leonardo.

Proposição 5 (Identidade de Catalan)

Para os números naturais $\mathrm{n}$ e $\mathrm{k}, \operatorname{com} \mathrm{n} \geq \mathrm{k}$, se $H L e_{n}$ é o n-ésimo número híbrido de Leonardo, então tem-se a seguinte identidade:

$$
\begin{aligned}
H L e_{n-k} H L e_{n+k}-H L e_{2}^{n}= & 4\left(H F_{n-k+1} H F_{n+k+1}-H F_{n+1}^{2}\right) \\
& -(1+i+\varepsilon+h)\left(2 H F_{n-k+1}+2 H F_{n+k+1}-4 H F_{n+1}\right) .
\end{aligned}
$$

Demonstração

$$
\begin{aligned}
& H L e_{n-k} H L e_{n+k}-H L e_{2}^{n}= {\left[2 H F_{n-k+1}-(1+i+\varepsilon+h)\right]\left[2 H F_{n+k+1}-(1+i+\varepsilon+h)\right] } \\
&-\left[2 H F_{n+1}-(1+i+\varepsilon+h)\right]^{2} \\
&=4 H F_{n-k+1} H F_{n+k+1}-2 H F_{n-k+1}(1+i+\varepsilon+h)-2 H F_{n+k+1}(1+i+\varepsilon+h) \\
&+(1+i+\varepsilon+h)^{2}-4 H F_{n+1}^{2}+4 H F_{n+1}(1+i+\varepsilon+h)-(1+i+\varepsilon+h \\
&=4 H F_{n-k+1} H F_{n+k+1}-(1+i+\varepsilon+h)\left(2 H F_{n-k+1}+2 H F_{n+k+1}-4 H F_{n+1}\right)-4 H F_{n+1}^{2} \\
&=4\left(H F_{n-k+1} H F_{n+k+1}-H F_{n+1}^{2}\right)-(1+i+\varepsilon+h)\left(2 H F_{n-k+1}+2 H F_{n+k+1}-4 H F_{n+1}\right)
\end{aligned}
$$

A identidade de Cassini é um caso particular da identidade de Catalan, quando $\mathrm{k}=1$ obtemos o seguinte resultado. 
Proposição 6 (Identidade de Cassini)

Para os números naturais $\mathrm{n}$, se $H L e_{n}$ é o n-ésimo número híbrido de Leonardo, então temos a seguinte identidade:

$$
\begin{aligned}
H L e_{n-1} H L e_{n+1}-H L e_{n}^{2}= & 4\left(H F_{n} H F_{n+2}-H F^{2}{ }_{n+1}\right. \\
& -(1+i+\varepsilon+h)\left(2 H F_{n}+2 H F_{n+2}-4 H F_{n+1}\right)
\end{aligned}
$$

\section{Demonstração}

$$
\begin{aligned}
H L e_{n-1} H L e_{n+1}-H L e^{2}= & {\left[2 H F_{n}-(1+i+\varepsilon+h)\right]\left[2 H F_{n+2}-(1+i+\varepsilon+h)\right] } \\
& -\left[H F_{n+1}-(1+i+\varepsilon+h)\right]^{2} \\
= & 4 H F_{n} H F_{n+2}-2 H F_{n}(1+i+\varepsilon+h)-2 H F_{n+2}(1+i+\varepsilon+h) \\
& +(1+i+\varepsilon+h)^{2}-4 H F^{2}{ }_{n+1}+4 H F_{n=1}(1+i+\varepsilon+h) \\
& -(1+i+\varepsilon+h)^{2} \\
= & 4 H F_{n} H F_{n+2}-(1+i+\varepsilon+h)\left(2 H F_{n}+2 H F_{n+2}-4 H F_{n+1}\right) \\
& -4 H F_{n+1}^{2} \\
= & 4\left(H F_{n} H F_{n+2}-H F^{2}{ }_{n+1}-(1+i+\varepsilon+h)\left(2 H F_{n}+2 H F_{n+2}\right.\right. \\
& \left.-4 H F_{n+1}\right)
\end{aligned}
$$

Proposição 7 (Identidade de d'Ocagne)

Sendo $\mathrm{n}$ um número inteiro positivo e m um número inteiro não negativo. Se $H L e_{n}$, é o n-ésimo número híbrido de Leonardo, a expressão da identidade de d'Ocagne é dada por:

$$
H L e_{m} H L e_{n+1}-H L e_{m+1} H L e_{n}=4\left(H F_{n} H F_{n+2}-H F_{n+1}^{2}\right)-(1+i+\varepsilon+h)\left(2 H F_{n}+2 H F_{n+2}-4 H F_{n+1}\right)
$$

\section{Demonstração}

Esta identidade é uma generalização da identidade de Cassini, fazendo $\mathrm{m}=$ $\mathrm{n}-1$, temos a seguinte identidade: 
$H L e_{m} H L e_{n+1}-H L e_{m+1} H L e_{n}=H L e_{n-1} H L e_{n+1}-H L e^{2}{ }_{n}$

A demonstração desta proposição se encontra na Proposição 6.

\subsection{Identidades}

Nesta seção, apresentaremos identidades em torno dos híbridos de Leonardo e identidades que relacionam os híbridos de Leonardo com os híbridos de Fibonacci.

Identidade 3.1.1

A soma dos n primeiros números dos híbridos de Leonardo é dada por:

$$
\sum_{j=0}^{n} H L e_{j}=H L e_{n+2}-[(n+1)(1+i+\varepsilon+h)+1] .
$$

\section{Demonstração}

Utilizando a Proposição 2 e Catarino et al. (2014), temos:

$$
\begin{aligned}
\sum_{j=0}^{n} H L e_{j} & =\sum_{j=0}^{n}\left[2 H F_{j+1}-(1+i+\varepsilon+h)\right] \\
& =2 \sum_{j=0}^{n} H F_{j+1}-\sum_{j}^{n}(1+i+\varepsilon+h) \\
& =2 \sum_{j=0}^{n} H F_{j+1}-(n+1)(1+i+\varepsilon+h) \\
& =2 \sum_{j=0}^{n+1} H F_{j}-(n+1)(1+i+\varepsilon+h) \\
& =2\left(H F_{n+2}+H F_{n+1}-1\right)-(n+1)(1+i+\varepsilon+h) \\
& =\left(2 H F_{n+3}-1\right)-[(n+1)(1+i+\varepsilon+h)+1] \\
& =H L e_{n+2}-[(n+1)(1+i+\varepsilon+h)+1] .
\end{aligned}
$$


Identidade 3.1 .2

A soma dos híbridos de Leonardo de índices 2n par por ser descrito por:

$$
\sum_{j=0}^{n} H L e_{2 j}=2 H F_{2 n+2}-(n+1)(1+i+\varepsilon+h)
$$

\section{Demonstração}

Utilizando a Proposição 2, temos:

$$
\begin{aligned}
\sum_{j=0}^{n} H L e_{2 j} & =\sum_{j=0}^{n}\left[2 H F_{2 j+1}-(1+i+\varepsilon+h)\right] \\
& =2 \sum_{j=1}^{n+1} H F_{2 j-1}-(\mathrm{n}+1)(1+i+\varepsilon+h) \\
& =2 H F_{2 n+2}-(n+1)(1+i+\varepsilon+h)
\end{aligned}
$$

Identidade 3.1.3

A soma dos híbridos de Leonardo de índices n ímpar por ser descrito por:

$$
\sum_{j=0}^{n} H L e_{2 j}=2 H F_{2 n+3}-(n+1)(1+i+\varepsilon+h)
$$

\section{Demonstração}

Utilizando a Proposição 2, temos:

$$
\begin{aligned}
\sum_{j=0}^{n} H L e_{2 j+1} & =\sum_{j=0}^{n}\left[2 H F_{2 j+2}-(1+i+\varepsilon+h)\right] \\
& =2 \sum_{j=0}^{n+1} H F_{2 j}-(\mathrm{n}+1)(1+i+\varepsilon+h) \\
& =2 H F_{2 n+3}-(n+1)(1+i+\varepsilon+h)
\end{aligned}
$$


Identidade 3.1.4

Para $\mathrm{n} \geq 0$, temos:

$$
\sum_{j=0}^{n}\left(H F_{j}+H L e_{j}\right)=H F_{n+2}+H L e_{n+2}-[(n+2)(1+i+\varepsilon+h)+1]
$$

\section{Demonstração}

Utilizando a relação de recorrência dos números híbridos de Fibonacci apresentado por Cerda-Morales (2018) e a Identidade 3.11, temos:

$$
\begin{aligned}
\sum_{j=0}^{n}\left(H F_{j}+H L e_{j}\right) & =\sum_{j=0}^{n} H F_{j}+\sum_{j=0}^{n} H L e_{j} \\
& =H F_{n+1}+H F_{n}-(1+i+\varepsilon+h)+H L e_{n+2}-[(n+1)(1+i+\varepsilon+h)+1] \\
& =H F_{n+2}+H L e_{n+2}-[(n+1)(1+i+\varepsilon+h)+1+(1+i+\varepsilon+h)] \\
& =H F_{n+2}+H L e_{n+2}-[(n+2)(1+i+\varepsilon+h)+1] .
\end{aligned}
$$

\section{CONCLUSÃO}

No presente trabalho introduzimos o conjunto dos números híbridos e a sequência de Leonardo, sequência esta que está relacionada à famosa sequência de Fibonacci . A partir destes dois conteúdos matemáticos preliminares, seguimos em torno do processo de hibridização dos números de Leonardo, na qual, foi exibido definições e propriedades inerentes aos números híbridos de Leonardo, sua relação de recorrência, função geradora, fórmula de Binet, representação matricial e identidades bastante conhecidas na matemática.

Para trabalhos futuros, pode-se investigar mais propriedades desse conteúdo, ou ainda, utilizar esse conteúdo voltado para o ensino criando situaçõesproblemas com o intuito de instigar o lado investigativo do estudante. Por fim, este artigo possibilita contribuir no âmbito matemático e oportunizar aos professores 
de Matemática conhecimento sobre o conjunto dos números híbridos, a sequência de Leonardo e seu processo evolutivo.

\section{REFERÊNCIAS}

Alves, F. R. V., Vieira, R. P. M. (2019). The newton fractal's Leonardo sequence study with the google colab. International Electronic Journal of Mathematics Education, 15(2), em0575.

Alves, F. R. V., Catarino, P. M. M. C., Vieira, R. P. M., Mangueira, M. C. d. S. (2020). Teaching recurrent sequences in Brazil using historical facts and graphical illustrations. Acta Didactica Napocensia, 13(1), 87-104.

Catarino, P. (2019). On k-Pell hybrid numbers. Journal of Discrete Mathematical Sciences and Cryptography, 22(1), 83-89.

Catarino, P., Vasco, P., Borges, A., Campos, H., Aires, A. (2014). Sums, products and identities involving k-Fibonacci and k-Lucas sequences. JP Journal of Algebra, Number Theory and Applications, 32(1), 63.

Catarino, P. M., Borges, A. (2020). On Leonardo numbers. Acta Mathematica Universitatis Comenianae, 89(1), 75-86.

Cerda-Morales, G. (2018). Investigation of generalized hybrid Fibonacci numbers and their properties. arXiv preprint arXiv:180602231.

Koshy, T. (2001). Fibonacci and Lucas Numbers with Applications, vol 1. New York: Wileyand Sons publications.

Mangueira, M. C. d. S., Alves, F. R. V., Catarino, P. M. M. C. (2020a). Números híbridos de Mersenne. CQD - Revista Eletrônica Paulista de Matemática, 18, 1-11.

Mangueira, M. C. d. S., Vieira, R. P. M., Alves, F. R. V., Catarino, P. M. M. C. (2020b). The hybrid numbers of Padovan and some identities. Em: Annales Mathematicae Silesianae, Sciendo, vol 34, pp. 256-267.

Mangueira, M. C. S., Alves, F. R. V. (2020). Números híbridos de Fibonacci e Pell. Revista Thema, 17(3), 831-842.

Özdemir, M. (2018). Introduction to hybrid numbers. Advances in Applied Clifford Algebras, 28(1), 11. Shannon, A. (2019). A note on generalized Leonardo numbers. Note on Number Theory and Discrete Mathematics, 25(3), 97-101.

Szynal-Liana, A. (2018). The Horadam hybrid numbers. Discussiones Mathematicae-General Algebra and Applications, 38(1), 91-98. 
Szynal-Liana, A., Włoch, I. (2019). On Jacobsthal and Jacobsthal-Lucas hybrid numbers. Em: Annales Mathematicae Silesianae, Sciendo, vol 33, pp. 276-283.

Vieira, R. P. M., Alves, F. R. V., Catarino, P. M. M. C. (2019). Relações bidimensionais e identidades da sequência de Leonardo. Revista Sergipana de Matemática e Educação Matemática, 4(2), 156-173.

Vieira, R. P. M., dos Santos Mangueira, M. C., Alves, F. R. V., Catarino, P. M. M. C. (2020). The matrix form of Leonardo's numbers. Ciência e Natura, 42, 1-13.

\section{Contribuições de Autoria}

\section{1 - Milena Carolina dos Santos Mangueira:}

Mestranda em Ensino de Ciências e Matemática, bolsista pela Coordenação de Aperfeiçoamento de Pessoal de Nível Superior - CAPES, Departamento de Matemática, Instituto Federal de Educação, Ciências e Tecnologia do Ceará https://orcid.org/0000-0002-4446-155X - milenacarolina24@gmail.com Contribuição: Conceituação, Investigação, Metodologia, Escrita - primeira redação, Escrita - revisão e edição

\section{2 - Francisco Regis Vieira Alves:}

Doutor em ensino de Matemática, bolsista de Produtividade do Conselho Nacional de Desenvolvimento Científico e Tecnológico - CNPq - PQ2, Professor do Doutorado em Associação em Rede de Pós-Graduação em Ensino (RENOEN), Departamento de Matemática, Instituto Federal de Educação, Ciências e Tecnologia do Ceará https://orcid.org/0000-0003-3710-1561 -fregiis@ifce.edu.br Contribuição: Conceituação, Investigação, Escrita - revisão e edição

\section{3 - Paula Maria Machado Cruz Catarino}

Doutora em Matemática, Professora Associado da UTAD, Departamento de Matemática da Escola de Ciências e Tecnologia Universidade de Trás-os-Montes e Alto Douro, Portugal

https://orcid.org/0000-0001-6917-5093 - pcatariino23@gmail.com

Contribuição: Conceituação, Investigação, Escrita - revisão e edição

\section{Como citar este artigo}

MANGUEIRA, M. C. S.; ALVES, F. R. V.; CATARINO, P. M. M. C.. Os números híbridos de Leonardo. Ciência e Natura, Santa Maria, v. 43, e82, p. 01-20, 2021. DOI 10.5902/2179460X63773. Disponível em: https://doi.org/10.5902/2179460X63773. Acesso em: dia, mês abreviado. ano. 